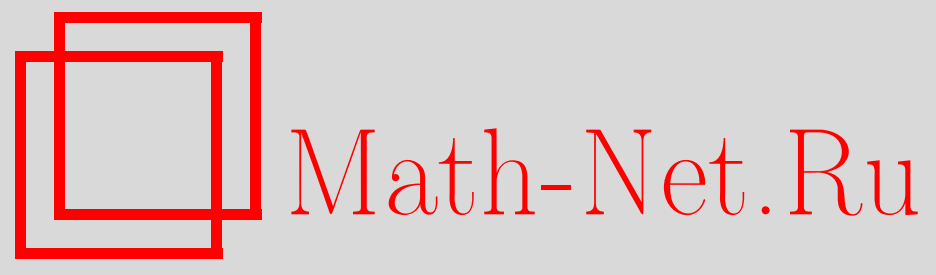

М. Я. Кельберт, Ю. М. Сухов, Асимптотическое поведение ветвящейся диффузии на гиперболическом пространстве, Теория вероятн. и ее примен., 2007, том 52, выпуск 4, 660-684

DOI: https://doi.org/10.4213/tvp1528

Использование Общероссийского математического портала MathNet.Ru подразумевает, что вы прочитали и согласны с пользовательским соглашением

http://www.mathnet.ru/rus/agreement

Параметры загрузки:

IP : 3.89 .185 .249

26 апреля 2023 г., 15:12:59

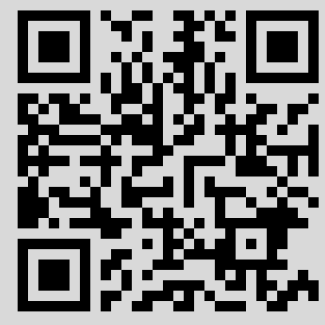




\title{
АСИМПТОТИЧЕСКОЕ ПОВЕДЕНИЕ ВЕТВЯЩЕЙСЯ ДИФФУЗИИ НА ГИПЕРБОЛИЧЕСКОМ ПРОСТРАНСТВЕ
}

\begin{abstract}
Рассматривается ветвяшийся диффузионный процесс на пространстве Лобачевского $\mathbf{H}^{d}$. Проблема состоит в оценке хаусдорфовой размерности предельного множества на границе (абсолюте) $\partial \mathbf{H}^{d}$. В случае однородной ветвящейся диффузии элегантная формула для хаусдорфовой размерности была получена С. Лалли и Т. Селке [5] при $d=2$ и Ф.И. Карпелевичем, Е. А. Печерским и Ю. М. Суховым [3] для обшего d. Затем М. Я. Кельберт и Ю. М. Сухов [4] распространили эту формулу на случай, когда ветвящаяся диффузия в некотором смысле асимптотически однородна (т.е. ее основной параметр, так называемый потенциал ветвления, стремится к постоянному предельному значению вблизи абсолюта). В этой статье доказывается, что хаусдорфова размерность предельного множества оценивается сверху и снизу в терминах максимальных и минимальных значений потенциала ветвления. Как и в [4], метод основан на свойствах минимальных положительных решений задачи Штурма-Лиувилля с обшим потенциалом и элементах гармонического анализа на $\mathbf{H}^{d}$. Мы исследуем связь хаусдорфовой размерности со свойствами рекуррентности и транзиентности (возвратности и невозвратности) ветвящейся диффузии, как они определены А. Григоряном и М.Я. Кельбертом [1] на общих римановых многообразиях.
\end{abstract}

Ключевые слова и фразы: гиперболическое пространство, возвратность, невозвратность, предельное множество, хаусдорфова размерность, орисферическая проекция, эллиптические уравнения с частными производными, задача Штурма-Лиувилля, минимальное положительное решение.

1. Введение. В этой статье изучаются геометрические аспекты асимптотического поведения ветвящихся диффузионных процессов на пространстве Лобачевского. Хотя ветвяшиеся диффузионные процессы (ВДП) довольно популярная модель в теории вероятностей и

* Mathematics Department, University of Wales Swansea, Singleton Park, Swansea, SA2 8PP, UK; e-mail: M. Kelbert@swansea.ac.uk

** Statistical Laboratory, DPMMS/CMS, University of Cambridge, Cambridge CB3 oWB, UK; e-mail: yms@statslab.cam.ac.uk 
ее приложениях, многие их асимптотические свойства остаются относительно неизученными. Мы следуем подходу, предложенному в работе [5], где однородный ветвяшийся диффузионный процесс (ОВДП), по-видимому, впервые рассматривался на плоскости Лобачевского $\mathbf{H}^{2}$ и обсуждались его асимптотические свойства. В частности, свойство возвратности означает, что с вероятностью 1 любой компакт посещается в сколь угодно большие моменты времени хотя бы одним потомком в ветвящемся процессе, а свойство невозвратности означает, что это происходит с вероятностью, меньшей 1. Возможно, наиболее интересным наблюдением, сделанным в [5], был тот факт, что для ОВДП случайное множество $\Lambda$ предельных точек на границе (абсолюте) $\partial \mathbf{H}^{2}$ имеет с вероятностью 1 детерминированное значение хаусдорфовой размерности, которое может быть вычислено явно.

Результаты работы [5] были перенесены в [3] на ОВДП на пространстве Лобачевского $\mathbf{H}^{d}$. Оказалось, что хаусдорфова размерность $h$ предельного множества $\Lambda$ на абсолюте $\partial \mathbf{H}^{d}$, как функция естественных параметров процесса, испытывает своеобразный «фазовый переход», а именно, она непрерывно возрастает от 0 до $(d-1) / 2$, а затем меняется скачком до $d-1$ (в этом случае предельное множество $\Lambda$ совпадает со всем абсолютом $\partial \mathbf{H}^{d}$ ). Это связано с деликатными спектральными свойствами генератора процесса на $\mathbf{H}^{d}$, который в случае ОВДП равен $-\Delta / 2$, где $\Delta$ - оператор Лапласа-Бельтрами. С вероятностной точки зрения важный факт состоит в том, что любая траектория ветвящейся диффузии на $\mathbf{H}^{d}$ экспоненциально быстро притягивается к случайной точке $w \in \partial \mathbf{H}^{d}$, равномерно распределенной на $\partial \mathbf{H}^{d}$, так что после некоторого случайного времени эта траектория не посещает любой выделенный компакт в $\mathbf{H}^{d}$. Однако если механизм ветвления является достаточно сильным (суперкритическим), то в процессе появляется столь большое число потомков, что некоторые из них могут (и будут) посещать этот компакт в сколь угодно большие моменты времени. Из этого факта выводится, что $\Lambda=\partial \mathbf{H}^{d}$ в суперкритическом случае.

В данной статье мы изучаем неоднородные ветвящиеся диффузионные процессы (НВДП) на $\mathbf{H}^{d}$. Некоторые вспомогательные результаты получены для НВДП на областях в $\mathbf{H}^{d}$ с индуцированной римановой метрикой. На первый взгляд представляется, что свойство однородности является критическим для всех доказательств в работах [5], [2] и [3]. С другой стороны, в недавней работе [4] было показано, что описанные выше результаты верны в случае «асимптотической» однородности ветвящегося процесса вблизи абсолюта. Поскольку хаусдорфова размерность является «локальной» характеристикой, можно ожидать, что она связана с поведением параметров ветвящегося процесса вблизи (соответствующим образом определяемых) экстремальных точек.

Указанное соображение и является исходной точкой настояшей ра- 
боты. Для корректного определения ветвящейся диффузии нужно задать закон индивидуального движения частиц и закон ветвления. В этой статье мы все еше предполагаем, что индивидуальное движение частиц является однородной диффузией на $\mathbf{H}^{d}$, и наш анализ связан со спектральными свойствами оператора Лапласа-Бельтрами. Однако механизм ветвления является совершенно общим. Он задается измеримой функцией $\lambda(z)$, определяющей интенсивность ветвления, и набором измеримых функций $p_{k}(z), k=1,2, \ldots$, определяющих вероятности получить $k$ потомков при ветвлении в точке $z \in \mathbf{H}^{d}$. Нижние и верхние оценки для хаусдорфовой размерности даются в терминах поведения функций $\lambda(z)$ и $p_{k}(z)$ вблизи экстремальных точек. Более точно, рассмотрим локальный потенциал ветвления (ЛПВ)

$$
V(z)=2 \lambda(z)(\kappa(z)-1)
$$

где $\kappa(z)$ - среднее число потомков при ветвлении в точке $z \in \mathbf{H}^{d}$ :

$$
\kappa(z)=\sum_{k \geqslant 0} k p_{k}(z)
$$

Хаусдорфова размерность предельного множества оценивается сверху и снизу в терминах, связанных с поведением функции $V(z)$ вблизи точек ее максимума и минимума.

В частности, суцествуют два типа локальных максимумов потенциала $V(z)$ : на границе $\partial \mathbf{H}^{d}$ и строго внутри $\mathbf{H}^{d}$ (т.е. вдали от границы), и между ними происходит «соревнование», в котором «приз»- это максимальное значение $h$ и тесно связанные с ним свойства возвратности или невозвратности. Интуитивная точка зрения, что максимум на абсолюте «более важен», чем внутри $\mathbf{H}^{d}$, обычно (но далеко не всегда) справедлива. Сложным является случай, когда максимальное значение $\mathrm{V}_{\mathrm{b}}^{+}$потенциала $V(z)$ на $\partial \mathbf{H}^{d}$ меньше, а максимальное значение $V_{\text {in }}^{+}$внутри $\mathbf{H}^{d}$ больше, чем наименьшее собственное значение оператора $-\Delta / 2$. В этом случае вероятностная интуиция недостаточна, и требуются детальные аналитические вычисления, результат которых и определяет ответ. С другой стороны, минимальные значения ЛПВ $V(z)$ появляются в нижней оценке хаусдорфовой размерности и не влияют на свойства возвратности и невозвратности.

$\mathrm{C}$ нашей точки зрения модель НВДП на $\mathbf{H}^{d}$ имеет серьезные преимущества (отмеченные уже в [5]), которые стоит использовать при асимптотическом исследовании моделей случайного роста. Эта модель имеет богатую аналитическую и геометрическую структуру, позволяюшую явно вычислять ее параметры. Аналогичная задача в отсутствие подобной структуры потребует тщательного (и трудного) качественного анализа, и с нашей точки зрения ответы, полученные в этой статье, могут облегчить такую работу. 
2. Основные понятия и результаты. Пространство Лобачевского $\mathbf{H}^{d}$ можно определить как внутренность единичного шара в $\mathbf{R}^{d}$, снабженную метрикой Клейна. (При $d=2$ обычно используют модель с метрикой Пуанкаре, а $\mathbf{H}^{2}$ представляется как внутренность круга или полуплоскость. Однако модели Клейна и Пуанкаре изоморфны.) Краткое изложение основных понятий, адресованное неспециалистам, можно найти в [3]. Метрика Клейна совместима с обычной евклидовой топологией (но не с евклидовой метрикой) в $\mathbf{R}^{d}$, и абсолют, т.е. бесконечно удаленная граница $\partial \mathbf{H}^{d}$ пространства $\mathbf{H}^{d}$, отвечает единичной сфере в $\mathbf{R}^{d}$. Снабдим абсолют обычной евклидовой топологией.

Геодезическая линия в $\mathbf{H}^{d}$ - это направленная хорда $\gamma$, соединяющая точки $w, w^{\prime} \in \partial \mathbf{H}^{d}$ (будем говорить, что $\gamma$ выходит из $w$ и входит в $\left.w^{\prime}\right)$. Она «канонически» изоморфна направленной прямой $\mathbf{R}$ с обычной евклидовой метрикой. Более точно, при канонической изометрии: a) начало координат на прямой $\mathbf{R}$ соответствует орисферической проекции $\pi^{\text {ho }}(0)$ центра $\left.0 \in \mathbf{H}^{d}, \sigma\right)$ гиперболическое расстояние между точками $z, z^{\prime} \in \gamma$ равно евклидову расстоянию между их образами на $\mathbf{R}$. Центр $0 \in \mathbf{H}^{d}$ играет роль точки отсчета и может быть заменен любой другой точкой; это не повлияет на формулировки наших теорем. С другой стороны, свобода выбора точки отсчета дает больше возможностей для проверки наложенных ниже условий и, таким образом, расширяет границы применимости результатов.

Семейство геодезических, исходящих из данной точки $w \in \partial \mathbf{H}^{d}$ (соответственно входящих в $\left.w^{\prime} \in \partial \mathbf{H}^{d}\right)$, образует геодезический пучок $\mathfrak{G}_{w}^{-} \mathrm{c}$ полюсом выхода $w$ (соответственно геодезический пучок $\mathfrak{G}_{w^{\prime}}^{+}$с полюсом входа $\left.w^{\prime}\right)$. гиперповерхность $\mathbf{S}_{w} \subset \mathbf{H}^{d}$, ортогональная геодезическим из пучка $\mathfrak{G}_{w}^{-}$, называется орисферой (с полюсом $w$ ); в метрике Клейна- это проколотая граница эллипсоида, касающегося абсолюта в точке $w$. При естественной стереографической проекции орисфера $\mathbf{S}_{w}$ изоморфна $\mathbf{R}^{d-1}$. Стереографическая проекция естественным образом продолжается на проколотый абсолют и тем самым порождает так называемую стереографическую метрику $\chi_{w}$ на $\partial \mathbf{H}^{d}$ с полюсом в $w$. Ясно, что эта метрика зависит от выбора точки $w$. (В модели Пуанкаре плоскости Лобачевского, где $\mathbf{H}^{2}$ представляется как верхняя полуплоскость $\mathbf{C}^{+}$, абсолют отвечает вещественной прямой $\mathbf{R} \subset \mathbf{C}$, и при $w=\infty$ метрика $\chi_{w}-$ это обычная евклидова метрика на $\mathbf{R}$.)

В дальнейшем, говоря о стереографической проекции, мы используем евклидовы термины, относяшиеся к орисфере $\mathbf{S}_{w}$ или абсолюту $\partial \mathbf{H}^{d}$ (например, говорим о $(d-1)$-мерном кубе, лежащем в $\mathbf{S}_{w}$ или $\left.\partial \mathbf{H}^{d}\right)$. Хаусдорфова размерность $h(\Lambda)$ предельного множества $\Lambda \subseteq \partial \mathbf{H}^{d}$ вычисляется в стереографической метрике $\chi_{w_{*}}$ для некоторой выбранной точки $w_{\star} \in \partial \mathbf{H}^{d}$. Выбор этой точки не влияет на утверждения приводимых ниже теорем и не будет далее обсуждаться. Более того, все резуль- 
таты остаются справедливыми и для другого хорошо известного типа метрик на $\partial \mathbf{H}^{d}$, так называемых угловых метрик. Угловая метрика $\varphi_{z}$ также зависит от выбора точки отсчета $z \in \mathbf{H}^{d}$; в этой метрике расстояние $\varphi_{z}\left(w, w^{\prime}\right)$ между точками $w, w^{\prime} \in \partial \mathbf{H}^{d}$ равно евклидову углу между геодезическими $\gamma$ и $\gamma^{\prime}$, выходящими из $w$ и $w^{\prime}$ соответственно и проходящими через $z$. (В модели Пуанкаре, где плоскость Лобачевского представляется открытым единичным кругом, абсолют - это единичная окружность $S^{1} \in \mathrm{C}$, и при $z=0$ метрика $\varphi_{z}$ - это евклидова метрика на $S^{1}$.)

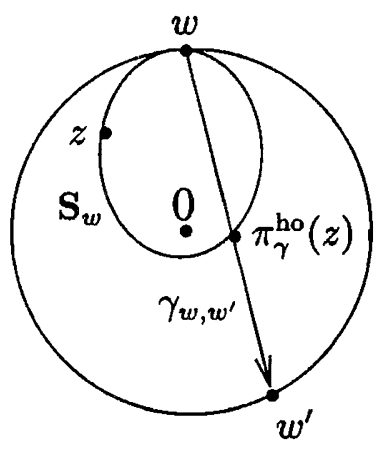

Puc. 1

Орисферы с полюсом в $w$ образуют слоение в пространстве $\mathbf{H}^{d}$; вместе с проколотым абсолютом $\partial \mathbf{H}^{d} \backslash\{w\}$ они образуют слоение в $\overline{\mathbf{H}}^{d} \backslash\{w\}$, где $\overline{\mathbf{H}}^{d}$ - это замкнутый единичный шар в $\mathbf{R}^{d}$. Открытый эллипсоид с границей $\mathbf{S}_{w}$ называется оришаром с полюсом $w$ и обозначается $\mathbf{B}\left(\mathbf{S}_{w}\right)$. См. рис. 1.

$\mathrm{B}$ дальнейшем мы опускаем индекс $d$ в обозначениях типа $\mathbf{H}^{d}$. Пусть $\gamma \in \mathfrak{G}_{w}^{-}$- геодезическая линия. Геодезическая проекция $\pi_{\gamma}^{\text {ho }}$ отображает точку $z \in \mathbf{H}_{\text {в }} \pi_{\gamma}^{\text {ho }}(z)$ - точку, в которой $\gamma$ пересекает орисферу $\mathbf{S}_{w}$, проходящую через $z$. Напомним, что при канонической изометрии между $\gamma$ и $\mathbf{R}$ образ $\pi_{\gamma}^{\text {ho }}(0)$ центра $0 \in \mathbf{H}$ совпадает с началом координат на $\mathbf{R}$. $\mathrm{B}$ дальнейшем мы многократно используем каноническую изометрию между прямой $\mathbf{R}$ и геодезической $\gamma$, не оговаривая этого каждый раз. В частности, это устанавливает, как определяются подмножества в $\mathbf{H}$, образами которых являются полубесконечные интервалы $\left(-\infty, \pi_{\gamma}^{\text {ho }}(z)\right)$ и $\left(\pi_{\gamma}^{\text {ho }}(z), \infty\right)$ на геодезической $\gamma$, где $z \in \mathbf{H}$.

Однородный гиперболический диффузионный процесс (ОГДП) на $\mathbf{H}$ - это марковский процесс с генератором $-\Delta / 2$, где $\Delta$ - оператор Лапласа-Бельтрами на Н. Важное свойство состоит в том, что для каждой геодезической $\gamma \in \mathfrak{G}_{w}^{-}$образом ОГДП при отображении $\pi_{\gamma}^{\text {ho }}$ является броуновское движение на $\mathbf{R}^{1}$ со сносом $-(d-1) / 2$ и единичной диффу- 
зией. Это придает точный смысл утверждению, что траектория ОГДП на $\mathbf{H}$ экспоненциально быстро притягивается к на орисфере при дуальной проекции является стандартное броуновское движение с нулевым сносом и матрицей диффузии, пропорциональной единичной матрице I.)

Теперь рассмотрим ВДП $\Theta_{z_{0}}$ на $\mathbf{H}$, начинаюшийся из точки $z_{0} \in \mathbf{H}$, в котором индивидуальное движение частиц задается ОГДП, а ветвление в точке $z \in \mathbf{H}$ имеет интенсивность $\lambda(z)$ и при этом $k$ потомков возникают с вероятностями $p_{k}(z), k=0,1, \ldots$. Движение и ветвление различных потомков независимы. Функции $\lambda(z)$ и $p_{k}(z)$ предполагаются борелевскими и определенными на замыкании $\overline{\mathbf{H}}=\mathbf{H} \cup \partial \mathbf{H}$ в топологии замкнутого единичного шара в $\mathbf{R}^{d}$. Для простоты мы в дальнейшем предполагаем, что $p_{0} \equiv 0$. С другой стороны, мы не накладываем условий глобальной непрерывности функций $\lambda$ и $p_{k}$, хотя это и упростило бы изложение. Глобальные условия непрерывности неестественны потому, что гиперболическая метрика на $\mathbf{H}$ является сингулярной по отношению к евклидовой метрике на $\overline{\mathbf{H}}$ вблизи абсолюта $\partial \mathbf{H}$. Однако в точках локального максимума потенциала ветвления $V$ функции $\lambda(z)$ и $p_{k}(z)$ предполагаются непрерывными.

Обозначим $P(z)$ распределение числа потомков и $\kappa(z)$ среднее число потомков в точке $z \in \overline{\mathbf{H}}$ (cp. с (1.2)). Напомним, что $V(z)$ обозначает ЛIIВ в точке $z \in \mathbf{H}$ (см. (1.1)). Вообще говоря, мы не исключаем случая, когда $\kappa(z)$ и, следовательно, $V(z)$ принимают значение $+\infty$.

Важную роль играют значения $V_{\mathrm{b}}^{+}, V_{\mathrm{gl}}^{+}$и $V_{\mathrm{b}}^{-}$. Значения $V_{\mathrm{b}}^{+}$и $V_{\mathrm{gl}}^{+}$ определяются формулами

$$
V_{\mathrm{b}}^{+}=\sup _{z \in \partial \mathbf{H}} V(z) \quad \text { и } \quad V_{\mathrm{gl}}^{+}=\sup _{z \in \overline{\mathbf{H}}} V(z) ;
$$

очевидным образом, $V_{\mathrm{gl}}^{+} \geqslant V_{\mathrm{b}}^{+}$. Далее,

$$
V_{\mathrm{b}}^{-}=\inf _{z \in \partial \mathbf{H}} V(z)
$$

Мы разбираем различные случаи взаимного расположения максимумов $V_{\mathrm{b}}^{+}$и $V_{\mathrm{gl}}^{+}$и поведения интенсивности ветвления $\lambda(z)$ и распределения $P(z)$ вблизи точек этих максимумов. Введем некоторые технические предположения. Символ $\preceq$ используется для стохастического порядка между вероятностными распределениями на $\left.\mathbf{Z}_{+}=\{0,1,2, \ldots\}\right)$ : $P^{(1)} \preceq P^{(2)}$ означает, что $\sum_{l \geqslant k} p_{l}^{(1)} \leqslant \sum_{l \geqslant k} p_{l}^{(2)}$ для всех $k \geqslant 1$. Будем говорить, что $w^{+} \in \partial \mathbf{H}$ есть точка локального максимума для пары $(\lambda, P)$, если существует евклидова окрестность $U=U\left(w^{+}\right) \subset \mathbf{R}^{d}$ точки $w^{+}$такая, что для всех $z \in U \cap \overline{\mathbf{H}}$

$$
\lambda(z) \leqslant \lambda\left(w^{+}\right) \quad \text { и } \quad P(z) \preceq P\left(w^{+}\right) .
$$


Будем говорить, что $w^{+} \in \partial \mathbf{H}$ - точка глобального максимума для пары $(\lambda, P)$, если (2.2) выполняется для всех $z \in \overline{\mathbf{H}}$. Аналогичным образом определяется точка $z^{+} \in \mathbf{H}$ локального или глобального максимума. Точки локального и глобального минимума задаются заменой неравенств на противоположные.

$\mathrm{C}$ другой стороны, $w^{-} \in \partial \mathbf{H}$ называется точкой орисферического минимума для $(\lambda, P)$, если для любого $\varepsilon \in(0,1)$ существуют точка $w(\varepsilon) \in \partial \mathbf{H}$ и орисфера $\mathbf{S}_{w(\varepsilon)} \in \mathfrak{G}_{w(\varepsilon)}^{-}$с полюсом $w(\varepsilon)$ такие, что для любого $z \in \mathbf{H} \backslash \mathbf{B}\left(\mathbf{S}_{w(\varepsilon)}\right)$

$$
\lambda(z) \geqslant \lambda\left(w^{-}\right)(1-\varepsilon) \quad \text { и } \quad p_{k}(z) \geqslant p_{k}\left(w^{-}\right)(1-\varepsilon) \quad \forall k \geqslant 2 .
$$

Наглядно это означает, что точки $z^{-} \in \mathbf{H}$ локального минимума для пары $(\lambda, P)$ не накапливаются на «массивном» подмножестве абсолюта $\partial \mathrm{H}$. Определение орисферического максимума дается аналогичным образом, с заменой неравенства на противоположное и $1-\varepsilon$ на $1+\varepsilon$. Соответственно оно означает, что точки локального максимума для пары $(\lambda, P)$ не накапливаются на «массивном» подмножестве абсолюта.

Далее, введем свойства непрерывности пары $(\lambda, P)$ в точках $w^{+}$ или $z^{+}$локального или глобального максимума. Будем говорить, что точка максимума $z^{+}$является точкой непрерывности (снизу) для $(\lambda, P)$, если для любого $\varepsilon \in(0,1)$ существует евклидова окрестность $O=$ $O_{\varepsilon}\left(z^{+}\right) \subset \mathbf{R}^{d}$ точки $z^{+}$такая, что для любого $z \in O \cap \overline{\mathbf{H}}$

$$
(1-\varepsilon) \lambda\left(z^{+}\right) \leqslant \lambda(z) \quad \text { и } \quad(1-\varepsilon) p_{k}\left(z^{+}\right) \leqslant p_{k}(z) \quad \forall k \geqslant 2 .
$$

Аналогичное определение дается для точки максимума $w^{+} \in \partial \mathbf{H}$. Различие между (2.3a) и $(2.3 \mathrm{~b})$ - в областях, где выполняются соответствующие неравенства. См. рис. 2.

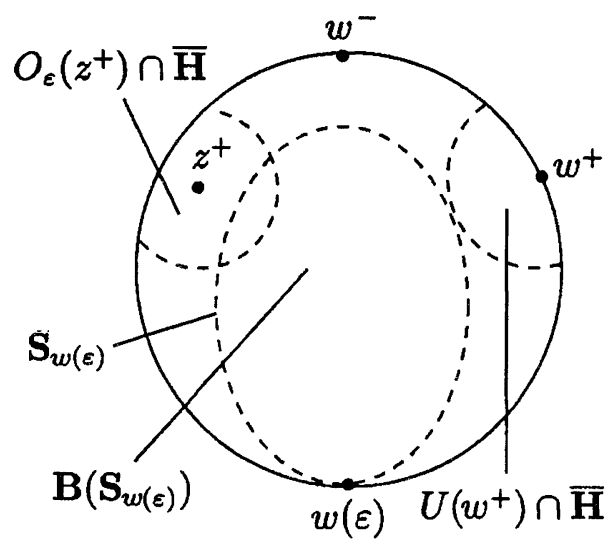

Рис. 2 
Мы рассматриваем следующие случаи.

Случай 1: $V_{\mathrm{b}}^{+}=V_{\mathrm{gl}}^{+}$. В этом случае мы предполагаем, что значение $V_{\mathrm{b}}^{+}$достигается в некоторой точке $w^{+} \in \partial \mathbf{H}$, которая является точкой глобального максимума и непрерывности для пары $(\lambda, P)$. Мы также предполагаем, что значение $V_{\mathrm{b}}^{-}$достигается в точке $w^{-} \in \partial \mathbf{H}$ орисферического минимума для $(\lambda, P)$.

Случай 1 можно разбить на два подслучая: 1A (более трудный), когда $V_{\mathrm{b}}^{+}=V_{\mathrm{gl}}^{+} \leqslant \frac{1}{8}(d-1)^{2}$, и 1Б (более простой), когда $V_{\mathrm{b}}^{+}=V_{\mathrm{gl}}^{+}>$ $\frac{1}{8}(d-1)^{2}$. Отметим, что принятая здесь форма технических условий обслуживает нужды проводимого ниже анализа.

Случай 2A: $V_{\mathrm{b}}^{+} \leqslant V_{\mathrm{gl}}^{+}<\frac{1}{8}(d-1)^{2}$. В этом случае мы предположим, что $V_{\mathrm{b}}^{+}$достигается в некоторой точке $w^{+} \in \partial \mathbf{H}$ локального и орисферического максимума и непрерывности для пары $(\lambda, P)$. Мы также предположим, что $V_{\mathrm{b}}^{-}$достигается в некоторой точке $w^{-} \in \partial \mathbf{H}$ локального и орисферического минимума для $(\lambda, P)$.

Случай 2Б: $V_{\mathrm{b}}^{+} \leqslant \frac{1}{8}(d-1)^{2}<V_{\mathrm{gl}}^{+}$. Чтобы подчеркнуть, что «больший» локальный максимум лежит внутри $\mathbf{H}$, положим $V_{\mathrm{gl}}^{+}=V_{\mathrm{in}}^{+}$. В этом случае мы предполагаем, что $V_{\mathrm{b}}^{+}$и $V_{\text {in }}^{+}$достигаются в некоторых точках $w^{+} \in \partial \mathbf{H}$ и $z^{+} \in \mathbf{H}$ непрерывности для пары $(\lambda, P)$ таких, что $w^{+}$является точкой локального, а $z^{+} \in \mathbf{H}$ - точкой глобального максимума для пары $(\lambda, P)$. Мы также предположим, как и выше, что $V_{\mathrm{b}}^{-}$достигается в точке орисферического минимума для $(\lambda, P)$.

Простым является случай $2 \mathrm{~B}$, когда $\frac{1}{8}(d-1)^{2}<V_{\mathrm{b}}^{-} \leqslant V_{\mathrm{b}}^{+} \leqslant V_{\mathrm{gl}}^{+}$. Здесь мы снова предположим, что $V_{\mathrm{b}}^{-}$достигается в некоторой точке глобального и орисферического минимума для $(\lambda, P)$.

Резюмируя, всюду ниже в этой статье мы предполагаем, что индивидуальное движение в процессе $\Theta_{z_{0}}$ задается однородной диффузией на $\mathbf{H}$, а интенсивность ветвления $\lambda(z)$ и распределение числа потомков $P(z)$ в точке $z \in \overline{\mathbf{H}}$ удовлетворяют одному из перечисленных выше условий. Обозначим $\mathbb{P}_{z_{0}}$ распределение вероятностей процесса $\Theta_{z_{0}}$. Пусть $\Lambda_{z_{0}}$ - случайное замкнутое множество на $\partial \mathbf{H}$, образованное предельными точками НВДП $\Theta_{z_{0}}$ в евклидовой топологии на $\overline{\mathbf{H}}$. Символ $h\left(z_{0}\right)$ обозначает хаусдорфову размерность множества $\Lambda_{z_{0}}$, вычисленную в некоторой фиксированной метрике, $\chi_{w_{\star}}$ или $\varphi_{z_{\star}}$, на $\partial \mathbf{H}$; это есть случайная величина, распределение которой зависит от $z_{0}$. Будем говорить, что процесс $\Theta_{z_{0}}$ возвратный, если для любого $t_{0}>0$ и любого открытого множества $O \subset \mathbf{H}$ с вероятностью 1 хотя бы один потомок начальной частицы попадет в $O$ при некотором $t>t_{0}$. Процесс $\Theta_{z_{0}}$ является невозвратным, если эта вероятность меньше 1 (в [1] даются необходимые и достаточные условия возвратности и невозвратности на римановых многообразиях). 
Теорема 1. А. В случае $1 \mathrm{~A}$ для любой начальной точки $z_{0} \in \mathbf{H}$ прочесс $\Theta_{z_{0}}$ является невозвратным и

$$
\begin{aligned}
\mathbb{P}_{z_{0}}\left\{\frac{1}{2}[d\right. & \left.-1-\sqrt{(d-1)^{2}-8 V_{\mathrm{b}}^{-}}\right] \\
& \left.\leqslant h\left(z_{0}\right) \leqslant \frac{1}{2}\left[d-1-\sqrt{(d-1)^{2}-8 V_{\mathrm{b}}^{+}}\right]\right\}=1 .
\end{aligned}
$$

Более того, для любого $z_{0} \in \mathbf{H}$

$$
\mathbb{P}_{z_{0}}\left\{\frac{1}{2}\left[d-1-\sqrt{(d-1)^{2}-8 V_{\mathrm{b}}^{+}}\right]-\varepsilon<h\left(z_{0}\right)\right\}>0 \quad \forall \varepsilon>0,
$$

т.е. верхняя гранича в (2.4) является точной.

Б. $B$ случае $1 \mathrm{Б}$ для любого $z_{0} \in \mathbf{H}$

$$
\mathbb{P}_{z_{0}}\left\{\Lambda_{z_{0}}=\partial \mathbf{H}\right\}>0
$$

m.е. $\Lambda_{z_{0}}$ c положительной вероятностью совпадает с дН. Более того, если $\mathbb{P}_{\bar{z}^{0}}\left\{\Lambda_{\bar{z}^{0}}=\partial \mathbf{H}\right\}=1$ для некоторого $\bar{z}^{0} \in \mathbf{H}$, mо для любого $z_{0} \in \mathbf{H}$ прочесс $\Theta_{z_{0}}$ является возвратньи $u \mathbb{P}_{z_{0}}\left\{\Lambda_{z_{0}}=\partial \mathbf{H}\right\}=1$.

Теорема 2. А. $B$ случае $2 \mathrm{~A}$ для любого $z_{0} \in \mathbf{H}$ прочесс $\Theta_{z_{\mathbf{0}}}$ является невозвратным и справедливы соотношения (2.4) $u$ (2.5).

Б. $B$ cлучае $2 \mathrm{5}$, как $и$ в теореме $1 . \mathrm{A}$, если $\mathbb{P}_{\bar{z}^{0}}\left\{\Lambda_{\bar{z}^{0}}=\partial \mathbf{H}\right\}=1$ для некоторого $\bar{z}^{0} \in \mathbf{H}$, то для любого $z_{0} \in \mathbf{H}$ прочесс $\Theta_{z_{0}}$ является возвратным и $\mathbb{P}_{z_{0}}\left\{\Lambda_{z_{0}}=\partial \mathbf{H}\right\}=1$.

В. $B$ случае $2 \mathrm{~B}$ прочесс $\Theta_{z_{0}}$ всегда является возвратным $u$ $\mathbb{P}_{z_{0}}\left\{\Lambda_{z_{0}}=\partial \mathbf{H}\right\}=1$.

Теорема 2.Б оставляет открытым вопрос о том, является ли процесс $\Theta_{z_{0}}$ возвратным или невозвратным, а также выполняются ли соотношения (2.4) и (2.6). Отметим, что условия (2.4) и (2.6) являются взаимоисключаюшими и, по-видимому, исчерпывают все возможности для предельного множества $\Lambda_{z_{0}}$ при весьма слабых ограничениях.

В теоремах 3.А и 3.Б (см. ниже) даются достаточные условия в случае 2Б для того, чтобы выполнялось соотношение (2.4) или (2.6). Эти условия формулируются в терминах функций бесселева типа $\mathscr{J}_{\nu}{ }_{\nu}$ и $\mathscr{J}_{\nu}{ }_{\nu}$ соответственно первого и второго рода с соответствующим образом выбранным индексом $\nu$. Более точно, $\mathscr{J}_{\nu}^{1}(y)=J_{i \nu}^{(1)}(i y)$ и $\mathscr{J}_{\nu}^{2}(y)=J_{i \nu}^{(2)}(i y)$, $y \in \mathbf{R}$, где $J_{\nu}^{(1)}$ и $J_{\nu}^{(2)}$ - стандартные функции Бесселя (см. [6]). Другими словами, $\mathscr{J}_{\nu}{ }_{\nu}$ и $\mathscr{J}_{\nu}{ }^{2}$ - решения уравнения типа Бесселя, имеющие специальные асимптотики при $y \sim 0: \mathscr{J}_{\nu}^{1}(y) \sim \operatorname{Re} y^{i \nu}$ и $\mathscr{J}_{\nu}^{2}(y) \sim \operatorname{Im} y^{i \nu}$. Обе функции $\mathscr{J}_{\nu}{ }_{\nu}$ и $\mathscr{J}_{\nu}{ }_{\nu}$ являются вещественными осциллирующими функциями вещественного аргумента.

В доказательствах теорем $3 . \mathrm{A}$ и 3.5 фигурируют множества $\mathscr{O}_{\nu}^{(1)}$ и $\mathscr{O}_{\nu}^{(2)}$ вещественных нулей функций $\mathscr{J}_{\nu}{ }_{\nu}(y)$ и $\mathscr{J}_{\nu}^{2}(y)$ соответственно. 
А именно, мы измеряем «массивность» максимумов ЛПВ $V(z)$, сравнивая их относительную позицию, высоту и ширину в терминах множеств $\mathscr{O}_{\nu}^{(1)}$ и $\mathscr{O}_{\nu}^{(2)}$. Более точно, результаты формулируются в терминах существования специальных верхних и нижних процессов, являющихся оценками ВДП и обладающих дополнительными свойствами симметрии.

Удобным понятием является локально орисферически однородная (ЛОО) пара $(\lambda, P)$. Здесь, как и ранее, $\lambda=\lambda(z)$ есть неотрицательная функция, а $P=P(z)-$ набор вероятностей, которые постоянны в конечном числе слоев между орисферами с фиксированным полюсом $w$. Точнее, пусть $\mathbf{S}_{w}^{(1)}, \mathbf{S}_{w}^{(2)}, \ldots, \mathbf{S}_{w}^{(2 \ell-1)}, \mathbf{S}_{w}^{(2 \ell)}$ - набор попарно различных орисфер с полюсом $w \in \partial \mathbf{H}$ таких, что $\mathbf{S}_{w}^{(i+1)}$ лежит внутри оришара $\mathbf{B}\left(\mathbf{S}_{w}^{(i)}\right)$, ограниченного орисферой $\mathbf{S}_{w}^{(i)}, i=1, \ldots, 2 \ell-1$. Обозначим $\mathbf{L}_{w}^{(j)}$ «орисферический слой» между $\mathbf{S}_{w}^{(2 j-1)}$ и $\mathbf{S}_{w}^{(2 j)}, j=1, \ldots, \ell$. Наконец, положим

$$
\mathbf{L}_{w}=\bigcup_{j=1}^{\ell} \mathbf{L}_{w}^{(j)}, \quad \mathbf{L}_{w}^{(0)}=\overline{\mathbf{H}} \backslash \mathbf{L}_{w}
$$

Орисферу $\mathbf{S}_{w}^{(i)}$ можно отнести к одному из примыкающих слоев или к $\mathbf{L}_{w}^{(0)}$. См. рис. 3.

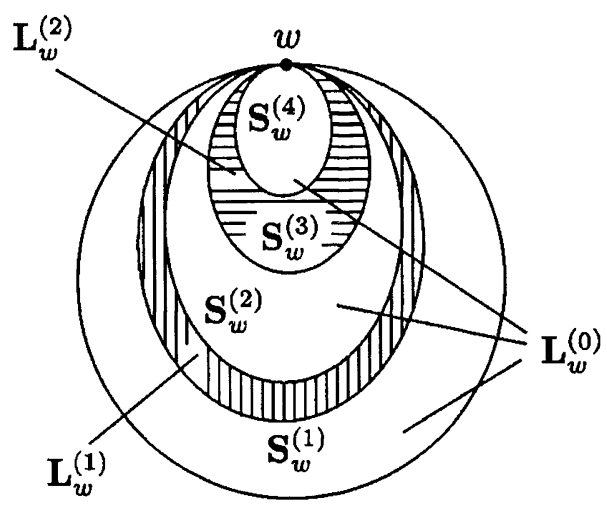

Puc. 3

Назовем $(\lambda, P)$ ЛОО-парой с полюсом $w \in \partial \mathbf{H}$ и орисферическими слоями $\mathbf{L}_{w}^{(1)}, \ldots, \mathbf{L}_{w}^{(\ell)}$, если: а) $\lambda(z)$ принимает постоянное значение $\lambda^{(j)}$ при $z \in \mathbf{L}_{w}^{(j)}$ и постоянное значение $\lambda^{(0)}$ при $z \in \mathbf{L}_{w}^{(0)}$, б) $P(z)$ совпадает с некоторым заданным распределением вероятностей $P^{(j)}$ при $z \in \mathbf{L}_{w}^{(j)}$ и с распределением вероятностей $P^{(0)}$ при $z \in \mathbf{L}_{w}^{(0)}$. Далее ЛОО-пара $(\bar{\lambda}(z), \bar{P}(z))$ со слоями $\mathrm{L}_{w}^{(1)}, \ldots, \mathbf{L}_{w}^{(\ell)}$ называется мажорантой для пары $(\lambda, P)$, если $\lambda(z) \leqslant \bar{\lambda}(z)$ и $P(z) \preceq \bar{P}(z)$ для любого $z \in \overline{\mathbf{H}}$. 
Другими словами, $\lambda(z) \leqslant \bar{\lambda}^{(j)}$ и $P(z) \preceq \bar{P}^{(j)}$ при $z \in \mathbf{L}_{w}^{(j)}, j=1, \ldots, \ell$, и $\lambda(z) \leqslant \bar{\lambda}^{(0)}$ и $P(z) \preceq \bar{P}^{(0)}$ при $z \in \mathrm{L}_{w}^{(0)}$.

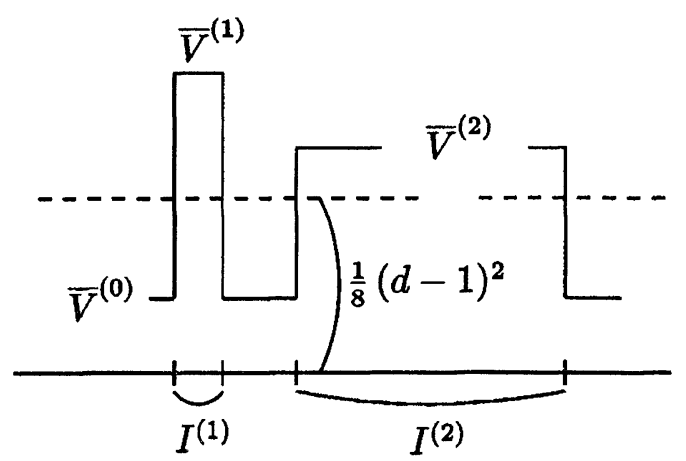

Рис. 4

Tеорема 3.А. $B$ случае 2Б предположим, ито пара $(\lambda(z), P(z))$ допускает ЛОО-мажоранту $(\bar{\lambda}(z), \bar{P}(z))$ с полюсом $\bar{w} \in \partial \mathbf{H}$ и слоями $\overline{\mathbf{L}}_{\bar{w}}^{(1)}, \ldots, \overline{\mathbf{L}}_{\bar{w}}^{(\ell)} u \overline{\mathbf{L}}_{\bar{w}}^{(0)}=\overline{\mathbf{H}} \backslash\left(\bigcup_{j=1}^{\ell} \overline{\mathbf{L}}_{\bar{w}}^{(j)}\right)$. Положим $\bar{\kappa}(z)=\sum_{k \geqslant 1} k \bar{p}_{k}(z)$. Пусть $\bar{\lambda}^{(j)}=\bar{\lambda}(z), \bar{P}(z)=\bar{P}^{(j)} u \bar{\kappa}(z)=\bar{\kappa}^{(j)}:=\sum_{k \geqslant 1} k \bar{p}^{(j)} n p u z \in \overline{\mathbf{L}}_{\bar{w}}^{(j)}$, $j=0,1, \ldots, l$. Зададим

$$
\bar{V}^{(j)}=2 \bar{\lambda}^{(j)}\left(\bar{\kappa}^{(j)}-1\right), \quad j=0,1, \ldots, \ell
$$

u предположим, что

$$
\bar{V}^{(0)} \leqslant \frac{(d-1)^{2}}{8} \quad u \quad \bar{V}^{(1)}, \ldots, \bar{V}^{(\ell)}>\frac{(d-1)^{2}}{8}
$$

(см. рис. 4.) Далее выберем геодезическую $\gamma \in \mathfrak{G}_{w}^{-}$и рассмотрим каноническую координату на $\gamma$. Пусть $I^{(j)}$ - это интервал прямой $\mathbf{R}$, соответствуюший пересечению $\gamma$ с $\mathbf{L}_{w}^{(j)}$. Предположим, что при всех $j=1, \ldots, \ell$ функиии бесселева типа $\mathscr{J}_{\bar{\nu}^{(j)}}^{1}(y)$ и $\mathscr{J}_{\bar{\nu}^{(j)}}^{2}(y)$ не имеют нулей в $I^{(j)}$. Тогда для всех $z_{0} \in \mathbf{H}$ прочесс $\Theta_{z_{0}}$ является невозвратным и выполняются свойства (2.4), (2.5).

В теореме 3.Б мы используем понятие орисферо-геодезического параллелепипеда (ОГП) и ОГП-миноранты $\left(\lambda^{-}(z), P^{-}(z)\right)$. Прежде всего определим геодезический конус $\mathbf{G}_{w, D}$ с полюсом в $w \in \partial \mathbf{H}$ и базой $D \subset \partial \mathbf{H} \backslash\{w\}$, где $D$ - открытый $(d-1)$-мерный куб (в стереографической проекции). Конус $\mathbf{G}_{w, D}$ состоит из всех геодезических $\gamma$ из пучка $\mathfrak{G}_{w}^{-}$, которые пересекают $\partial \mathbf{H}$ в точках $\widetilde{w} \in D$. Определим ОГП (с полюсом $w$ и базой $D$ ) как множество $П \subset \mathbf{H}$ между двумя различными орисферами $\mathbf{S}_{w}^{(1)}$ и $\mathbf{S}_{w}^{(2)}$ с полюсом $w \in \partial \mathbf{H}$, которое является пересечением соответствующего орисферического слоя $\mathbf{L}_{w}^{(1)}$ и геодезического конуса $\mathbf{G}_{w, D}$. См. рис. 5 . 


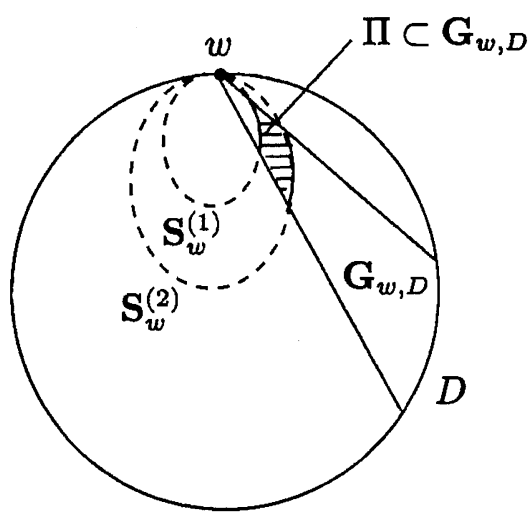

Puc. 5

Далее ОГП-пара (для ОГП П) - это пара $(\lambda(z), P(z))$ такая, что функция $\lambda(z)$ и распределение вероятности $P(z)$ остаются постоянными на П и на дополнении $\overline{\mathbf{H}} \backslash \Pi$. Иными словами, $\lambda(z)=\lambda_{\text {in }}$ и $P(z)=P_{\text {in }}$ при $z \in \Pi$ и $\lambda(z)=\lambda_{\text {out и }} P(z)=P_{\text {out }}$ при $z \in \overline{\mathbf{H}} \backslash \Pi$, где $\lambda_{\text {in }}$ и $\lambda_{\text {out }}-$ две заданные константы, а $P_{\text {in }}$ и $P_{\text {out }}$ - два заданных распределения вероятностей.

Наконец, ОГП-пара $(\underline{\lambda}(z), \underline{P}(z))$ называется ОГП-минорантой для пары $(\lambda(z), P(z))$, если $\underline{\lambda}(z) \leqslant \lambda(z)$ и $\underline{P}(z) \preceq P(z)$ для любого $z \in \overline{\mathbf{H}}$. Другими словами, $\underline{\lambda}_{\text {in }} \leqslant \lambda(z)$ и $\underline{P}_{\text {in }} \preceq P(z)$ при $z \in \Pi$, и $\underline{\lambda}_{\text {out }} \leqslant \lambda(z)$ и $\underline{P}_{\text {out }} \preceq P(z)$ при $z \in \overline{\mathbf{H}} \backslash$ П. Для определенности будем говорить, что $(\underline{\lambda}(z), \underline{P}(z))$ является ОГП-минорантой на ОГП П с полюсом $w$.

Теорема 3.Б. $\quad$ В случае 2Б предположим, что пара $(\lambda(z), P(z))$ допускает ОГП-миноранту $(\underline{\lambda}(z), \underline{P}(z))$ на ОГП П с полюсом $\underline{w} \in \partial \mathbf{H}$. Положим $\underline{\kappa}_{\text {in }}=\sum_{k} k \underline{p}_{k}(z) u \underline{V}_{\text {in }}=2 \underline{\lambda}_{\text {in }}\left(\underline{\kappa}_{\text {in }}-1\right)$ и предположим, что $\underline{\mathrm{in}}_{\mathrm{in}}>\frac{1}{8}(d-1)^{2}$ (см. pис. 6). Положим тажже

$$
\underline{\nu}_{\text {in }}=\sqrt{\underline{V}_{\text {in }}-\frac{1}{8}(d-1)^{2}} .
$$

Далее, зафиксируем геодезическую линию $\gamma \in \mathfrak{G}_{\underline{w}}^{-} c \gamma \cap \Pi \neq \varnothing и, \kappa а к u$ в теореме 3.А, рассмотрим каноническую координату на $\gamma$. Пусть $I^{(1)}$ - это интервал прямой $\mathbf{R}$, соответствующий пересечению $\gamma$ и П. Предположим, что каждая из функиий бесселева типа $\mathscr{J}_{\underline{v}_{\text {in }}}^{1}(y)$ $u \mathscr{J}_{\underline{v}_{\text {in }}}^{2}(y)$ имеет по меньшей мере два нуля в $I^{(1)}$. Тогда для каждого $z_{0} \in \mathbf{H}$ справедлива оченка (2.6). Более того, как и в теоремах 1.A u 2.Б, если $\mathbb{P}_{\bar{z}^{0}}\left\{\Lambda_{\bar{z}^{0}}=\partial \mathbf{H}\right\}=1$ для некоторого $\bar{z}^{0} \in \mathbf{H}$, то для любого $z_{0} \in \mathbf{H}$ проиесс $\Theta_{z_{0}}$ возвратен $и \mathbb{P}_{z_{0}}\left\{\Lambda_{z_{0}}=\partial \mathbf{H}\right\}=1$. 


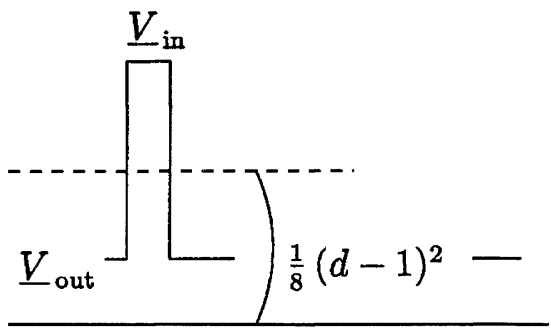

Pис. 6

3 а м е ч а н и е 2.1. В отличие от теоремы 3.А, теорема 3.Б применима и в случае неограниченного ЛПВ $V(z)$.

3 а м е ч а н и е 2.2. Как отмечалось ранее, теоремы 3.А и 3.Б остаются справедливыми, когда начало отсчета, определяюшее канонические координаты на геодезических линиях, переносится из 0 в произвольную точку $z^{\star} \in \mathbf{H}$. Другими словами, в теореме 3.Б играет роль только положение интервалов $I^{(1)}, \ldots, I^{(\ell)}$ относительно друг друга.

3. Логарифмические асимптотики решений эллиптических уравнений с частными производными и связанных с ними задач Штурма-Лиувилля. Доказательство оценки (2.5) основано на следующем аналитическом результате.

Лемма 3.1. Пусть $B=(-l,+\infty) \times D \subset \mathbf{R}^{d}-$ полубесконечная призма в $\mathbf{R}^{d}$, где $D-$ открытый куб в $\mathbf{R}^{d-1}$ :

$$
D=\left\{\underline{x}=\left(x_{1}, \ldots, x_{d-1}\right):\left|x_{1}\right|<\varkappa, \ldots,\left|x_{d-1}\right|<\varkappa\right\} .
$$

Зафиксируем $b>0$ и зададим функцию

$$
\phi(\underline{x})=\prod_{j=1}^{d-1} \cos \left(\frac{\pi}{2 \varkappa} x_{j}\right), \quad \underline{x}=\left(x_{1}, \ldots, x_{d-1}\right) \in D .
$$

Рассмотрим следуюшую эллиптическую граничную задачу для Функиии $B \ni(y, \underline{x}) \mapsto u(y ; \underline{x})$ :

$$
\begin{aligned}
& \frac{1}{2}\left[\frac{\partial^{2} u}{\partial y^{2}}(y ; \underline{x})+e^{2 b y} \sum_{j=1}^{d-1} \frac{\partial^{2} u}{\partial x_{j}^{2}}(y ; \underline{x})\right] \\
& -\frac{d-1}{2} \frac{\partial u}{\partial y}(y ; \underline{x})+v(y) u(y ; \underline{x})=0
\end{aligned}
$$

с граничным условием

$$
\left.u(y ; \underline{x})\right|_{\underline{x} \in \partial D}=0
$$


u потенииалом $v$ вида

$$
v(y)= \begin{cases}v_{0}, & -l<y<\ln a, \\ v_{1}, & y \geqslant \ln a\end{cases}
$$

2дe $a>e^{-l} u$

$$
0 \leqslant v_{0}, v_{1} \leqslant \frac{(d-1)^{2}}{8}
$$

Наконеи, пусть $u^{0}(y ; \underline{x})\left(=u_{l, \varkappa, a, b, v_{0}, v_{1}}^{0}(y ; \underline{x})\right)$ обозначает минимальное положительное речение задачи (3.2)-(3.4). Тогда для всех $y \in \mathbf{R}$ $u \underline{x} \in D$

$$
\lim _{l \rightarrow \infty} \frac{\ln u^{0}(y ; \underline{x})}{l}=\frac{d-1}{2}-\sqrt{\frac{(d-1)^{2}}{4}-2 v_{0}} .
$$

Лемма 3.2. В условиях леммы 3.1 предположим, что вместо (3.5) справедливо неравенство

$$
\max \left[v_{0}, v_{1}\right]>\frac{(d-1)^{2}}{8}
$$

Тогда для всех достаточно больших $l$ задача (3.2)-(3.4) не имеет положительных решений.

Лемма 3.3. Рассмотрим случай, где $V_{\mathrm{b}}^{+}<V_{\mathrm{gl}}^{+} \leqslant \frac{1}{8}(d-1)^{2}$. Обозначим $w^{0}(y)$ минимальное положительное решение следующей задачи на $(-l, \infty)$ :

$$
\frac{1}{2} w^{\prime \prime}(y)-\frac{(d-1)}{2} w^{\prime}(y)+v(y) w(y)=0
$$

с граничным условием

$$
w(-l)=1
$$

Предположим, что

$$
v(y)= \begin{cases}V_{\mathrm{b}}^{+}+\varepsilon, & -l<y<\ln a, \\ V_{\mathrm{gl}}^{+}, & y \geqslant \ln a,\end{cases}
$$

где $a>e^{-l}$. Тогда для любого $y \in \mathbf{R}$ и при достаточно малом $\varepsilon>0$

$$
\lim _{l \rightarrow \infty} \frac{\ln w^{0}(y)}{l}=\frac{d-1}{2}-\sqrt{\frac{(d-1)^{2}}{4}-2\left(V_{\mathrm{b}}^{+}+\varepsilon\right)} .
$$

3 а м е ч а н и е 3.1. Задача (3.8)-(3.9) может рассматриваться как аналог задачи Штурма-Лиувилля с условием минимальности, играющим роль второго граничного условия. 
Лемма 3.4. Рассмотрим задачу (3.8)-(3.9), где потенииал $v(y)$ принимает $\ell+1$ значение, $\ell \geqslant 1$. Более точно, пусть $e^{-l}<a_{1}<b_{1}<$ $a_{2}<b_{2}<\cdots<a_{\ell-1}<b_{\ell-1}$. Положим

$$
v(y)=\left\{\begin{array}{cll}
\frac{V_{\mathrm{b}}^{+}+\varepsilon,}{(d-1)^{2}} & -l<y<\ln a_{1}, & \\
8 & \ln a_{j} \leqslant y<\ln b_{j}, & j=1, \ldots, \ell-1, \\
V_{j}^{+}, & \ln b_{j} \leqslant y<\ln a_{j+1}, & j=1, \ldots, \ell-2, \\
\frac{(d-1)^{2}}{8}, & y>\ln b_{\ell-1}, &
\end{array}\right.
$$

аде $V_{\mathbf{b}}^{+}+\varepsilon \leqslant \frac{1}{8}(d-1)^{2} \leqslant V_{j}^{+}, j=1, \ldots, \ell-1$. Предположим, ито интервал $\left(\ln b_{j}, \ln a_{j+1}\right)$ не пересекается с объединением $\mathscr{O}_{\bar{\nu}_{j}}^{(1)} \cup \mathscr{O}_{\bar{\nu}_{j}}^{(2)}$. Здесь $\mathscr{O}_{\bar{\nu}_{j}}^{(1)}$ и $\mathscr{O}_{\bar{\nu}_{j}}^{(2)}$ - множества вещественных нулей функций бесселева типа $\mathscr{J}_{\bar{\nu}_{j}}(y)=J_{i \bar{\nu}_{j}}^{(1)}(i y)$ u $\mathscr{J}_{\bar{\nu}_{j}}^{2}(y)=J_{i \bar{\nu}_{j}}^{(2)}(i y)$ с индексом $\bar{\nu}_{j}, j=1, \ldots, \ell-1$ соответственно, где

$$
\bar{\nu}_{j}=\sqrt{V_{j}^{+}-\frac{(d-1)^{2}}{8}} .
$$

Тогда

$$
\lim _{l \rightarrow \infty} \frac{\ln w^{0}(y)}{l}=\frac{d-1}{2}-\sqrt{\frac{(d-1)^{2}}{4}-2\left(V_{\mathrm{b}}^{+}+\varepsilon\right)} .
$$

для всех $y \in \mathbf{R}$ и достаточно мальх $\varepsilon>0$.

Лемма 3.5. Рассмотрим задачу (3.2)-(3.4) с потенииалом $v(y)=$ $v(y, \underline{x})$ вида

$$
v(y)= \begin{cases}V_{\mathrm{b}}^{+}-\varepsilon, & -l<y<\ln a, \\ 0, & \ln a \leqslant y<\ln b, \\ V_{\mathrm{gl}}^{+}-\varepsilon, & \ln b \leqslant y<\ln c, \\ 0, & y \geqslant \ln c,\end{cases}
$$

где $V_{\mathrm{b}}^{+} \leqslant \frac{1}{8}(d-1)^{2}<V_{\mathrm{gl}}^{+}$. Предположим, что интервал $(\ln b, \ln c)$ содержит пару последовательных нулей каждой из функиий бесселева типа $\mathscr{J}_{\underline{\nu}}{ }^{1}(y)=J_{i \underline{\nu}}^{(1)}(i y)$ u $\mathscr{J}_{\underline{\nu}}^{2}(y)=J_{i \underline{\nu}}^{(2)}(i y)$ с индексом

$$
\underline{\nu}(=\underline{\nu}(\varepsilon))=\sqrt{V_{\mathrm{g} 1}^{+}-\varepsilon-\frac{(d-1)^{2}}{8}} .
$$

Тогда для достаточно малого $\varepsilon>0$ и достаточно большого $l$ задача (3.2)-(3.4) с потенциалом вида (3.14) не имеет неотричательных решений. Более того, задача (3.8)-(3.9) с потенииалом (3.14) также не имеет неотричательных решений при достаточно мальх $\varepsilon>0$ и достаточно больших $l$. 
Доказательства лемм 3.1-3.5 аналогичны друг другу. По этой причине мы разберем в деталях только доказательство леммы 3.1 , после чего прокомментируем изменения, которые потребуются для доказательства леммы 3.2. Тогда изменения, необходимые для доказательства других лемм, станут очевидными.

Д ок азательст во ле м мы 3.1. Будем искать решение в виде

$$
u(y ; \underline{x})=w(y) e^{(d-1) y / 2} \prod_{j=1}^{d-1} \cos \left(\frac{\pi}{2 \varkappa} x_{j}\right) .
$$

Тогда для функции $w(y)$ мы получим задачу

$$
\frac{1}{2} w^{\prime \prime}(y)+\left[v(y)-\frac{(d-1)^{2}}{8}-\frac{\pi^{2}(d-1)}{8 \varkappa^{2}} e^{2 b y}\right] w(y)=0, \quad y>-l,
$$

с граничным условием

$$
w(-l)=1
$$

Затем, переходя к экспоненциальной шкале по $y$, где

$$
t=\frac{\pi \sqrt{d-1}}{2 b \varkappa} e^{b y}, \quad g(t)=w(y)
$$

получаем уравнение

$$
t^{2} g^{\prime \prime}(t)+t g^{\prime}(t)-\left[b^{-2} \nu^{2}(y(t))+t^{2}\right] g(t)=0, \quad t>\frac{\pi \sqrt{d-1}}{2 b \varkappa} e^{-b l}
$$

с граничным условием

$$
g\left(\frac{\pi \sqrt{d-1}}{2 b \varkappa} e^{-b l}\right)=e^{(d-1) l / 2} .
$$

Функция $\nu^{2}(\tau)$ задается соотношением

$$
\nu^{2}(\tau)=\frac{(d-1)^{2}}{4}-2 v(y(\tau)), \quad \tau \in \mathbf{R},
$$

где $y(\tau)=b^{-1}[\ln (b \tau)-\ln (\pi \sqrt{d-1} /(2 \varkappa))]$. Это завершает подготовительный этап доказательства леммы 3.1 .

Теперь мы воспользуемся предположением, что $v$ принимает два значения, см. (3.4). Таким образом, вместо (3.20) возникают два уравнения

$$
\begin{gathered}
t^{2} g^{\prime \prime}(t)+t g^{\prime}(t)-\left[b^{-2} \nu_{0}^{2}+t^{2}\right] g(t)=0, \\
\frac{\pi \sqrt{d-1}}{2 b \varkappa} e^{-b l}<t<\frac{\pi \sqrt{d-1} a^{b}}{2 b \varkappa}
\end{gathered}
$$


и

$$
\begin{gathered}
t^{2} g^{\prime \prime}(t)+t g^{\prime}(t)-\left[b^{-2} \nu_{1}^{2}+t^{2}\right] g(t)=0 \\
t \geqslant \frac{\pi \sqrt{d-1} a^{b}}{2 b \varkappa}
\end{gathered}
$$

Здесь

$$
\nu_{0}^{2}=\frac{(d-1)^{2}}{4}-2 v_{0}, \quad \nu_{1}^{2}=\frac{(d-1)^{2}}{4}-2 v_{1} \text {. }
$$

Каждое из уравнений (3.23) и (3.24) есть хорошо известное дифференциальное уравнение Кельвина (Томсона) с индексами $\nu_{0}>0$ и $\nu_{1}>0$ соответственно (см. [6, с. 77]). Общие решения уравнений (3.23) и (3.24) имеют вид

$$
g_{0}(t)=C_{0} K_{\nu_{0} / b}^{(1)}(t)+D_{0} K_{\nu_{0} / b}^{(2)}(t), \quad g_{1}(t)=C_{1} K_{\nu_{1} / b}^{(1)}(t)+D_{1} K_{\nu_{1} / b}^{(2)}(t)
$$

соответственно, здесь $K_{\nu}^{(1)}$ и $K_{\nu}^{(2)}$ обозначают функции Кельвина индекса $\nu$ и рода 1 и 2 соответственно, а $C_{0}, D_{0}, C_{1}$ и $D_{1}$ суть произвольные константы.

Хорошо известно, что при $t \rightarrow 0+$ функция $K_{\nu}^{(1)}(t)$ стремится к нулю, а функция $K^{(2)}(t)$ имеет двойственную сингулярность:

$$
K_{\nu}^{(1)}(t) \sim \alpha_{\nu}^{(1)} t^{\nu}, \quad K_{\nu}^{(2)}(t) \sim \frac{\alpha_{\nu}^{(2)}}{t^{\nu}}
$$

где $\alpha_{\nu}^{(1)}$ и $\alpha_{\nu}^{(2)}$ - некоторые (известные) положительные константы. Таким образом, произвольное решение уравнения (3.20) в случае потенциала $v$, принимающего два значения $v_{0}$ и $v_{1}$, получается с помощью условий «склейки» функций $(3.26)$ в точке $t=\pi \sqrt{d-1} a^{b} /(2 b \varkappa)$ (нам необходимо гарантировать непрерывность решения и его производной). Это дает два уравнения на параметры $C_{0}, D_{0}, C_{1}$ и $D_{1}$, что отвечает сокращению числа степеней свободы с четырех до двух.

Соответственно, общее решение уравнения (3.20) имеет вид

$$
\begin{array}{r}
w(y ; p)=C_{0} e^{(d-1) y / 2} K_{\nu_{0} / b}^{(1)}\left(p \frac{e^{b y}}{b}\right)+D_{0} e^{(d-1) y / 2} K_{\nu_{0} / b}^{(2)}\left(p \frac{e^{b y}}{b}\right) \\
\text { при }-l<y<\ln a, \\
w(y ; p)=C_{1} e^{(d-1) y / 2} K_{\nu_{1} / b}^{(1)}\left(p \frac{e^{b y}}{b}\right)+D_{1} e^{(d-1) y / 2} K_{\nu_{1} / b}^{(2)}\left(p \frac{e^{b y}}{b}\right) \\
\text { при } \quad \ln a<y .
\end{array}
$$

Здесь $p=\pi \sqrt{d-1} /(2 \varkappa)$, а $C_{0}(p), D_{0}(p), C_{1}(p)$ и $D_{1}(p)$ выбираются так, чтобы функция $w(y)$ принадлежала классу $\mathrm{C}^{1}$ при $y=\ln a$. 
Теперь напомним, что мы ишем минимальное положительное решение задачи (3.2)-(3.4). Отсюда немедленно следует, что $C_{1}=0$. Остается найти $C_{0}, D_{0}$ и $D_{1}$ из трех линейных уравнений

$$
\begin{aligned}
& C_{0} K_{\nu_{0} / b}^{(1)}\left(p \frac{a^{b}}{b}\right)+D_{0} K_{\nu_{0} / b}^{(2)}\left(p \frac{a^{b}}{b}\right)=D_{1} K_{\nu_{1} / b}^{(2)}\left(p \frac{a^{b}}{b}\right), \\
& C_{0}\left[K_{\nu_{0} / b-1}^{(1)}\left(p \frac{a^{b}}{b}\right)-K_{\nu_{0} / b+1}^{(1)}\left(p \frac{a^{b}}{b}\right)\right] \\
& \quad+D_{0}\left[K_{\nu_{0} / b-1}^{(2)}\left(p \frac{a^{b}}{b}\right)-K_{\nu_{0} / b+1}^{(2)}\left(p \frac{a^{b}}{b}\right)\right] \\
& =D_{1}\left[K_{\nu_{1} / b-1}^{(2)}\left(p \frac{a^{b}}{b}\right)-K_{\nu_{1} / b+1}^{(2)}\left(p \frac{a^{b}}{b}\right)\right]
\end{aligned}
$$

и

$$
C_{0} K_{\nu_{0} / b}^{(1)}\left(\frac{p}{b} e^{-b l}\right)+D_{0} K_{\nu_{0} / b}^{(2)}\left(\frac{p}{b} e^{-b l}\right)=e^{(d-1) l / 2} .
$$

Отсюда получаем:

$$
\begin{aligned}
& D_{0}=e^{(d-1) l / 2}\left[\widehat{K}_{20}+\widehat{K}_{10} \frac{\left(K_{20}^{\text {left }}-K_{20}^{\text {right }}\right) K_{21}-K_{20}\left(K_{21}^{\text {left }}-K_{21}^{\text {right }}\right)}{K_{10}\left(K_{21}^{\text {left }}-K_{21}^{\text {right }}\right)-K_{21}\left(K_{10}^{\text {left }}-K_{10}^{\text {right }}\right)}\right]^{-1} \\
& C_{0}=D_{0}(p) \frac{\left(K_{20}^{\text {left }}-K_{20}^{\text {right }}\right) K_{21}-\left(K_{21}^{\text {left }}-K_{21}^{\text {right }}\right) K_{20}}{\left(K_{21}^{\text {left }}-K_{21}^{\text {right }}\right) K_{10}-\left(K_{10}^{\text {left }}-K_{10}^{\text {right }}\right) K_{21}}
\end{aligned}
$$

и

$$
D_{1}=\frac{1}{K_{21}}\left(K_{20} D_{0}+K_{10} C_{0}\right) \text {. }
$$

Здесь

$$
\begin{aligned}
\widehat{K}_{20} & =K_{\nu_{0} / b}^{(2)}\left(\frac{p}{b} e^{-b l}\right), & \widehat{K}_{10} & =K_{\nu_{0} / b}^{(1)}\left(\frac{p}{b} e^{-b l}\right), \\
K_{20}^{\text {left }} & =K_{\nu_{0} / b-1}^{(2)}\left(p \frac{a^{b}}{b}\right), & K_{20}^{\text {right }} & =K_{\nu_{0} / b+1}^{(2)}\left(p \frac{a^{b}}{b}\right), \\
K_{21} & =K_{\nu_{1} / b}^{(2)}\left(p \frac{a^{b}}{b}\right), & K_{20} & =K_{\nu_{0} / b}^{(2)}\left(p \frac{a^{b}}{b}\right), \\
K_{21}^{\text {left }} & =K_{\nu_{1} / b-1}^{(2)}\left(p \frac{a^{b}}{b}\right), & K_{21}^{\text {right }} & =K_{\nu_{1}+1}^{(2)}\left(p \frac{a^{b}}{b}\right), \\
K_{10} & =K_{\nu_{0} / b}^{(1)}\left(p \frac{a^{b}}{b}\right), & K_{11} & =K_{\nu_{1} / b}^{(1)}\left(p \frac{a^{b}}{b}\right), \\
K_{11}^{\text {left }} & =K_{\nu_{1} / b-1}^{(1)}\left(p \frac{a^{b}}{b}\right), & K_{11}^{\mathrm{right}} & =K_{\nu_{1} / b+1}^{(1)}\left(p \frac{a^{b}}{b}\right), \\
K_{10}^{\text {left }} & =K_{\nu_{0} / b-1}^{(1)}\left(p \frac{a^{b}}{b}\right), & K_{10}^{\mathrm{right}} & =K_{\nu_{0} / b+1}^{(1)}\left(p \frac{a^{b}}{b}\right) .
\end{aligned}
$$


Определив константы $C_{0}, D_{0}$ и $D_{1}$, мы можем задать функцию $w(y ; p)$ класса $\mathrm{C}^{1}$ по формулам (3.28). После этого минимальное положительное решение $u^{0}(y ; \underline{x})$ задачи (3.2)-(3.4) находится с помощью (3.16).

Заключительная часть доказательства леммы 3.1 состоит в вычислении логарифмической асимптотики функции $u^{0}(y ; \underline{x})$ при $l \rightarrow \infty$. Эта асимптотика непосредственным образом получается из приведенных выше явных формул. Лемма 3.1 доказана.

Д ок азат ел ь с т в о л е м м ы 3.2. Как отмечалось ранее, в доказательстве леммы 3.2 применимы те же аргументы, что и при доказательстве леммы 3.1. Единственное отличие состоит в том, что, скажем, при $v_{0}>\frac{1}{8}(d-1)^{2}$ решение для $-l<y<\ln a$ представляется как линейная комбинация функций типа Бесселя $\mathscr{J}_{\nu_{0} / b}^{1}(y)=J_{i \nu_{0} / b}^{(1)}(i y)$ и $\mathscr{J}_{\nu_{0} / b}^{2}(y)=J_{i \nu_{0} / b}^{(2)}(i y)$ первого и второго рода с индексом $\nu_{0} / b$, где $\nu_{0}^{2}=v_{0}-\frac{1}{8}(d-1)^{2}$, вместо соответствующих функций Кельвина. Тем самым, если $l$ превосходит максимальное расстояние между нулями функций бесселева типа $\mathscr{J}_{\nu_{0} / b}^{1}(y)$ и $\mathscr{J}_{\nu_{0} / b}^{2}(y)$ с индексом $\nu_{0} / b$, то любое решение осциллирует в направлении координаты $y$. Аналогичные аргументы применимы и в случае $v_{1}>\frac{1}{8}(d-1)^{2}$. Лемма 3.2 доказана.

Изменения, необходимые для проведения доказательств лемм 3.3-3.5, теперь очевидны.

\section{4. Вероятностная часть доказательств теорем.}

Доказательст в о т е о е мы 1.А. Начнем с доказательства формулы (2.4). Мы построим две вспомогательные пары, одна из которых является мажорантой, а другая - минорантой для исходной пары $(\lambda(z), P(z))$. (Более точно, мы работаем с фиксированной мажорантой и семейством минорант, зависящих от параметра $\varepsilon>0$.)

Мажорантная пара $\left(\lambda^{+}, P^{+}\right)$имеет постоянную интенсивность ветвления и фиксированное распределение числа потомков:

$$
\lambda^{+}=\lambda\left(w^{+}\right) \quad \text { и } \quad P^{+}=P\left(w^{+}\right) .
$$

При заданной начальной точке $z_{0} \in \mathbf{H}$ она порождает ОВДП $\Theta_{z_{0}}^{+}$на $\mathbf{H}$, мажорирующий исходный процесс $\Theta_{z_{0}}$. Легко видеть, что в случае $1 \mathrm{~A}$ «верхний» процесс $\Theta_{z_{0}}^{+}$является невозвратным. Таким образом, и процесс $\Theta_{z_{0}}$ является невозвратным при любой начальной точке $z_{0} \in \mathbf{H}$.

Семейство минорант $\left(\lambda^{- \text {,ho }}(\varepsilon), P^{- \text {,ho }}(\varepsilon)\right)$ строится следуюшим образом. При $\varepsilon \in(0,1)$ мы полагаем: для любого $z \in \mathbf{H} \backslash \mathbf{B}\left(\mathbf{S}_{w(\varepsilon)}\right)$

$$
\begin{aligned}
& {\left[\lambda^{- \text {, ho }}(\varepsilon)\right](z)=\lambda\left(w^{-}\right)(1-\varepsilon) \quad \text { и }} \\
& {\left[p_{k}^{-, \text {ho }}(\varepsilon)\right](z)=p_{k}\left(w^{-}\right)(1-\varepsilon) \quad \forall k \geqslant 2}
\end{aligned}
$$

и для любого $z \in \mathbf{B}\left(\mathbf{S}_{w(\varepsilon)}\right)$

$$
\left[\lambda^{-, \text {ho }}(\varepsilon)\right](z)=0 \quad \text { и } \quad\left[p_{k}^{-, \text {ho }}(\varepsilon)\right](z)=0 \quad \forall k \geqslant 2 .
$$


Эти миноранты определены корректно в силу того, что $w^{-}$есть точка орисферического минимума. По ним строятся «нижние» ветвяциеся диффузионные процессы $\Theta_{z_{0}}^{-}$, ho $(\varepsilon)$, являюшиеся НВДП на $\mathbf{H}$.

Для верхних и нижних процессов справедливы очевидные соотношения

$$
\mathbb{P}_{z_{0}}^{\text {joint }}\left(\Lambda_{z_{0}}^{-, \text {ho }}(\varepsilon) \subseteq \Lambda_{z_{0}} \subseteq \Lambda_{z_{0}}^{+}\right)=1
$$

из которых вытекает, что

$$
\mathbb{P}_{z_{0}}^{\text {joint }}\left(h^{-, \text {ho }}\left(z_{0} ; \varepsilon\right) \leqslant h\left(z_{0}\right) \leqslant h^{+}\right)=1 .
$$

Здесь $\mathbb{P}_{z_{0}}^{\text {joint }}$ обозначает совместное распределение процессов $\Theta_{z_{0}}^{- \text {, ho }}(\varepsilon), \Theta_{z_{0}}$ и $\Theta_{z_{0}}^{+}$, возникающее при их очевидной склейке. Далее, $\Lambda_{z_{0}}^{-, \text {ho }}(\varepsilon) \subset \partial \mathbf{H}-$ это предельное множество для $\Theta_{z_{0}}^{-, \text {ho }}(\varepsilon), \Lambda_{z_{0}}$ - предельное множество для исходного процесса $\Theta_{z_{0}}$, а $\Lambda_{z_{0}}^{+}$- предельное множество для процесса $\Theta_{z_{0}}^{+}$. Соответственно $h^{-, \text {ho }}\left(z_{0} ; \varepsilon\right), h\left(z_{0}\right)$ и $h^{+}$мы обозначаем их хаусдорфовы размерности. Мы знаем, что

$$
h^{+}= \begin{cases}\frac{1}{2}\left[d-1-\sqrt{(d-1)^{2}-8 V_{\mathrm{b}}^{+}}\right] & \text {в случае } 1 \mathrm{~A}, \\ d-1 & \text { в случае } 1 \mathrm{Б} .\end{cases}
$$

Формула (2.4) есть следствие вышеприведенной конструкции. Действительно, в случае $1 \mathrm{~A}$

$$
\mathbb{P}_{z_{0}}\left\{h\left(z_{0}\right) \leqslant \frac{1}{2}\left[d-1-\sqrt{(d-1)^{2}-8 V_{\mathrm{b}}^{+}}\right]\right\}=1 \text {. }
$$

С другой стороны, к процессу $\Theta_{z_{0}}^{-, \text {ho }}(\varepsilon)$ применимы результаты работы [4], откуда вытекает, что

$$
\mathbb{P}_{z_{0}}\left\{\frac{1}{2}\left[d-1-\sqrt{(d-1)^{2}-8 V_{\mathrm{b}}^{-}(1-\varepsilon)^{2}}\right]=h^{-, \text {ho }}\left(z_{0} ; \varepsilon\right)\right\}=1 \text {. }
$$

Переходя к пределу при $\varepsilon \rightarrow 0$, получаем

$$
\mathbb{P}_{z_{0}}\left\{\frac{1}{2}\left[d-1-\sqrt{(d-1)^{2}-8 V_{\mathrm{b}}^{-}}\right] \leqslant h\left(z_{0}\right)\right\}=1
$$

что завершает вывод формулы (2.4).

Для проверки соотношения (2.5) используется еше одно семейство минорант $\left(\lambda^{-}\left(z_{0} ; \varepsilon\right), P^{-}\left(z_{0} ; \varepsilon\right)\right)$, зависяших от $\varepsilon>0$ и начальной точки $z_{0}$. Эти миноранты строятся следующим образом. Введем понятие орисферо-геодезической трубки (ОГТ), прилегающей к точке $w^{+}$. Для этого выберем точку $w^{\prime} \in \partial \mathbf{H}, w^{\prime} \neq w^{+}$, и открытый $(d-1)$-мерный куб $D \subset \partial \mathbf{H} \backslash\left\{w^{\prime}\right\}$. Как и выше, рассмотрим конус $\mathbf{G}_{w^{\prime}, D}$ и орисферу $\mathbf{S}_{w^{\prime}} \in \mathfrak{G}_{w^{\prime}}^{-}$. ОГТ, прилегающая к $w^{+}$(и с полюсом $w^{\prime}$ и базой $D$ ), определяется как теоретико-множественная разность $\mathbf{G}_{w^{\prime}, D} \backslash \mathbf{B}\left(\mathbf{S}_{w^{\prime}}\right)$. 
Далее, при достаточно малом $\varepsilon>0$ рассмотрим ОГТ $\Gamma\left(z_{0} ; \varepsilon\right)$, прилегаюшую к $w^{+}$, содержащую точку $z_{0}$ и такую, что для любого $z \in \Gamma\left(z_{0} ; \varepsilon\right)$

$$
\lambda(z) \geqslant \lambda\left(w^{+}\right)(1-\varepsilon) \quad \text { и } \quad p_{k}(z) \geqslant p_{k}\left(w^{+}\right)(1-\varepsilon) \quad \forall k \geqslant 2 .
$$

Затем положим для любого $z \in \Gamma\left(z_{0} ; \varepsilon\right)$

$$
\begin{aligned}
& {\left[\lambda^{-}\left(z_{0} ; \varepsilon\right)\right](z)=\lambda\left(w^{+}\right)(1-\varepsilon) \quad \text { и }} \\
& {\left[p_{k}^{-}\left(z_{0} ; \varepsilon\right)\right](z)=p_{k}\left(w^{+}\right)(1-\varepsilon) \quad \forall k \geqslant 2}
\end{aligned}
$$

и для любого $z \in \mathbf{G}_{w^{\prime}, D} \backslash \Gamma\left(z_{0} ; \varepsilon\right)$

$$
\left[\lambda^{-}\left(z_{0} ; \varepsilon\right)\right](z)=0 \quad \text { и } \quad\left[p_{k}^{-}\left(z_{0} ; \varepsilon\right)\right](z)=0 \quad \forall k \geqslant 2 .
$$

См. pис. 7.

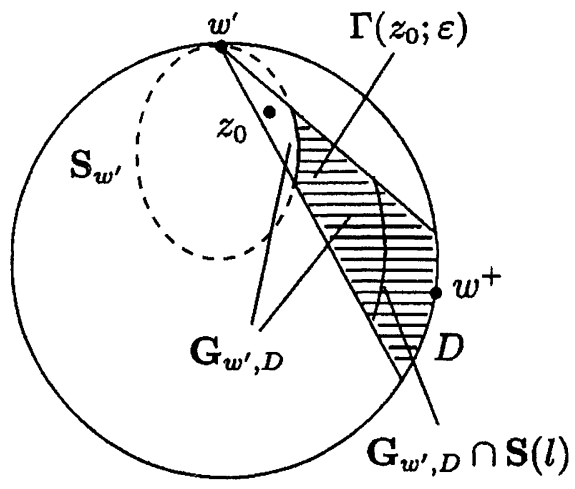

Puc. 7

Отметим, что база $D$ трубки $\Gamma\left(z_{0} ; \varepsilon\right)$ выбирается в зависимости от $\varepsilon$, тогда как полюс $w^{\prime}$ выбирается в зависимости от $z_{0}$ и $\varepsilon$.

Миноранта $\left(\lambda^{-}\left(z_{0} ; \varepsilon\right), P^{-}\left(z_{0} ; \varepsilon\right)\right)$ порождает нижний НВДП $\Theta_{z_{0}}^{-}(\varepsilon)$. Процесс $\Theta_{z_{0}}^{-}(\varepsilon)$ «живет» во внутренности конуса $\mathbf{G}_{w^{\prime}, D}$; частицы, достигающие границы $\partial \mathbf{G}_{w^{\prime}, D}$, «замораживаются» навсегда. (Это означает, что генератор $-\Delta / 2$ индивидуального диффузионного движения частицы включает условие Дирихле на $\left.\partial \mathbf{G}_{w^{\prime}, D} \cdot\right)$ Как и в $(4.3),(4.4)$, мы получаем, что

$$
\mathbb{P}_{z_{0}}^{\text {joint }}\left\{\Lambda_{z_{0}}^{-}(\varepsilon) \subseteq \Lambda_{z_{0}}\right\}=1
$$

откуда

$$
\mathbb{P}_{z_{0}}^{\text {joint }}\left(h^{-}\left(z_{0} ; \varepsilon\right) \leqslant h\left(z_{0}\right)\right)=1 .
$$

Здесь $\mathbb{P}_{z_{0}}^{\text {joint }}$ обозначает совместное распределение процессов $\Theta_{z_{0}}^{-}(\varepsilon)$ и $\Theta_{z_{0}}$, $\Lambda_{z_{0}}^{-}(\varepsilon)$ - предельное множество для $\Theta_{z_{0}}^{-}(\varepsilon)$, a $h^{-}\left(z_{0} ; \varepsilon\right)$ - его хаусдорфову размерность. 
Чтобы упростить изложение, мы будем часто опускать аргумент или индекс $z_{0}$, а также аргумент $\varepsilon$ в обозначениях $\Theta_{z_{0}}^{-}(\varepsilon), \Lambda_{z_{0}}^{-}(\varepsilon)$ и $h^{-}\left(z_{0} ; \varepsilon\right)$. Наша цель здесь - проверить, что в случае $1 \mathrm{~A}$ для любого $z_{0} \in \mathbf{H}$ сушествуют $\varepsilon^{0}=\varepsilon^{0}\left(z_{0}\right)>0$ и $c=c\left(z_{0}\right) \in(0,1)$ такие, что для любого $0<\varepsilon<\varepsilon^{0}$

$$
\mathbb{P}_{z_{0}}\left\{h^{-}\left(z_{0} ; \varepsilon\right) \geqslant \frac{1}{2}\left[d-1-\sqrt{(d-1)^{2}-8 V_{\mathrm{b}}^{+}(1-\varepsilon)^{2}}\right]\right\} \geqslant c .
$$

Отсюда при $\varepsilon \rightarrow 0$ получаем (2.5).

Ключевым шагом в доказательстве неравенства (4.10) является приводимая ниже лемма 4.1. При заданном $l>0$ рассмотрим модификацию $\widehat{\Theta}^{-}(l)$ нижнего процесса $\Theta^{-}$. А именно, выберем некоторую геодезическую $\gamma$ из пучка $\mathfrak{G}_{w^{\prime}}^{-}$(т.е. исходящую из $w^{\prime}$ ). Рассмотрим орисферу $\mathbf{S}(l)=\mathbf{S}_{w^{\prime}}(l)$ такую, что точка пересечения $\gamma$ и $\mathbf{S}(l)$ соответствует точке $l$ на $\mathbf{R}$ при каноническом изометрии. Возьмем $l$ достаточно большим таким, чтобы $z_{0}$ попадало в оришар $\mathbf{B}(\mathbf{S}(l))$. В модифицированном процессе $\widehat{\Theta}^{-}(l)$ частицы замораживаются на $(d-1)$-мерном кубе $\mathbf{G}_{w^{\prime}, D} \cap \mathbf{S}(l)$; cм. рис. 7. Как и в [5, предложение 1], нас интересует среднее число $\widehat{n}_{l}^{-}\left(z_{0}\right)$ частиц, достигающих $\mathbf{G}_{w^{\prime}, D} \cap \mathbf{S}(l)$ в процессе $\widehat{\Theta}^{-}(l)$ на бесконечном интервале времени:

$\widehat{n}_{l}^{-}\left(z_{0}\right)=\mathbb{E}_{z_{0}}\left(\right.$ число частиц в $\widehat{\Theta}^{-}(l)$, замороженных на $\left.\mathbf{G}_{w^{\prime}, D} \cap \mathbf{S}(l)\right)$.

Мы утверждаем, что при достаточно малом $\varkappa>0$

$$
\widehat{n}_{l}^{-}\left(z_{0}\right) \geqslant u_{1}^{0}\left(z_{0}\right), \quad z_{0} \in \mathbf{G}_{w^{\prime}, D},
$$

где функция $z_{0} \mapsto u_{1}^{0}\left(z_{0}\right)$ дает минимальное положительное решение задачи (3.2)-(3.4) в открытой области $B=B_{\varkappa}$ из леммы 3.1 (при соответствующей идентификации $\left.z_{0} \leftrightarrows(y, \underline{x})\right)$. См. (3.1a). Это утверждение справедливо, поскольку $u_{1}^{0}\left(z_{0}\right)$ задает среднее число частиц, замороженных на $D=D_{\varkappa}$, которые принимаются во внимание с вероятностями $\phi(x)$; см. (3.1b). В свою очередь, из последнего факта и леммы 3.1 вытекает, что

$$
\liminf _{l \rightarrow \infty} \frac{1}{l} \ln \widehat{n}_{l}^{-}\left(z_{0}\right) \geqslant \frac{1}{2}\left[d-1-\sqrt{(d-1)^{2}-8 V_{\mathrm{b}}^{+}(1-\varepsilon)^{2}}\right] .
$$

Приводимые ниже леммы 4.1 и 4.2 по сушеству мало отличаются от аналогичных утверждений из [5]. Ввиду этого, мы ограничимся набросками их доказательств.

Лемма 4.1. При достаточно малом $\varepsilon>0$ существует константа $c_{1}=c_{1}\left(z_{0}, \varepsilon\right) \in(0,1)$ такая, что хаусдорфова размерность $h^{-}\left(z_{0}, \varepsilon\right)$ в прочессе $\Theta_{z_{0}}^{-}(\varepsilon)$ удовлетворяет неравенству

$$
\mathbb{P}_{z_{0}}\left\{h^{-}\left(z_{0}, \varepsilon\right) \geqslant \liminf _{l \rightarrow \infty} \frac{1}{l} \ln \widehat{n}_{l}^{-}\left(z_{0}\right)-\varepsilon\right\} \geqslant c_{1} .
$$


Д ок аз а т ел ь с т о л е м м ы 4.1. Равенство (4.14) вытекает из леммы 3.1 и того факта, что $\widehat{n}_{l}^{-}\left(z_{0}\right) \geqslant u_{l}^{0}\left(z_{0}\right), z_{0} \in \mathbf{G}_{w^{\prime}, D}$, где $z_{0} \mapsto u_{1}^{0}\left(z_{0}\right)$ дает минимальное положительное решение задачи (3.2)-(3.4) (с идентификацией $\left.z_{0} \leftrightarrows(y, \underline{x})\right)$. Доказательство основано на конструкции замороженных/размороженных частиц, достигающих $(d-1)$ мерного куба $\mathbf{G}_{w^{\prime}, D} \cap \mathbf{S}(l)$, которая описана в разделе 5 работы [5] (см. также раздел 5 работы [3]). Здесь требуется очевидная модификация, так как наши ВДП $\widehat{\Theta}^{-}$и $\widehat{\Theta}^{-}(l)$ могут выродиться. Поэтому события вероятности 1, возникающие в [5], заменяются событиями положительной вероятности. Заметим, что, как и в [5] и [3], ключевую роль в этой конструкции играет лемма Фростмана.

Доказательст в о т е ре мы 1.Б. Основу доказательства составляет следуюшее свойство процесса $\Theta_{z_{0}}$ :

$\mathbb{P}_{z_{0}}\{$ существует компактная область $O \subset \mathbf{H}$, посещаемая

процессом $\Theta_{z_{0}}$ в как угодно большие моменты времени $\}>0$. (4.15)

Вывести равенство (2.6) из (4.15) можно, следуя аргументам, использованным в [5] (см. [5, предложения 9 и 10 и следствие 6]).

Более точно, при выполнении события в левой части (4.15) каждая точка $w \in \partial \mathbf{H}$ принадлежит множеству $\Lambda_{z_{0}}$. В самом деле, вероятность достичь произвольной окрестности данной точки $w \in \partial \mathbf{H}$ из $O$ положительна. При условии, что точки процесса $\Theta_{z_{0}}^{-}$посещают область $O$ в сколь угодно большие времена, будет предпринято счетное число таких попыток, и хотя бы одна из них окажется удачной.

Чтобы проверить равенства (4.15), мы вновь используем нижний ВДП $\Theta_{z_{0}}^{-}(\varepsilon)$ и его модификацию $\widehat{\Theta}^{-}(l)$ (см. выше), где $\varepsilon>0$ выбирается так, что

$$
V_{\mathrm{b}}^{+}(1-\varepsilon)^{2}>\frac{(d-1)^{2}}{8} .
$$

Равенство (4.15) легко вывести из следующей леммы.

Лемма 4.2. При любом $z_{0} \in \mathbf{H}$ существуют $\varepsilon>0$ и геодезический конус $\mathbf{G}_{w^{\prime}, D}$ такие, что а) выполняется оченка (4.16), б) $z_{0} \in \mathbf{G}_{w^{\prime}, D}$, в) выполняются оченки (4.7) uг чающий паре $\left(\lambda^{-}\left(z_{0} ; \varepsilon\right), P^{-}\left(z_{0} ; \varepsilon\right)\right)$ (см. (4.8a)), (4.8b)), удовлетворяет условию

$\mathbb{P}_{z_{0}}\left\{\right.$ существует компакт $O \subset \mathbf{G}_{w^{\prime}, D}$, посещаемый прочессом $\Theta_{z_{0}}^{-}(\varepsilon)$ в сколь угодно большие времена $\}>0$.

Д ок азат ел ь с т о ле м м 4 4.2. Доказательство основывается на следующих наблюдениях 1)-5).

1) Свойства а), б) и в) вытекают из предположений теоремы 1.Б. 
В оставшейся части доказательства мы анализируем свойства нижнего ВДП $\Theta^{-}=\Theta_{z_{0}}^{-}(\varepsilon)$ и его модификации $\widehat{\Theta}^{-}(l)$.

2) В соответствии с леммой 3.2 , для любого $\varkappa>0$ задача (3.2)-(3.4) с $v_{0}=V_{\mathrm{b}}^{+}(1-\varepsilon)^{2}$ и $v_{1}=0$ при достаточно больших $l$ (зависяших от $\varepsilon$ и $\varkappa)$ не имеет положительного решения.

3) Таким образом, в модифицированном процессе $\widehat{\Theta}^{-}(l)$ при достаточно больших $l$ среднее число $\widehat{n}_{l}^{-}\left(z_{0}\right)$ частиц, замороженных на орисфере $\mathbf{S}(l)$, равно $+\infty$.

4) Тем самым, в процессе $\Theta^{-}$при любом выборе его параметров среднее число $n_{\varepsilon, \varkappa, l}^{-}$частиц, достигающих $\mathbf{S}_{l}$, равно $+\infty$, снова при достаточно больших $l$.

5) Отсюда вытекает, что сушествует компакт $O \subset \mathbf{G}_{w, D}$ такой, что выполняется (4.15). Доказательство последнего факта повторяет аргументы, используемые в доказательстве следствия 3 из [5]. Лемма 4.2 доказана.

Д ок а з а т е ль с т в о т е о ре м ы 2.А. В этом доказательстве используется новое семейство мажорант $\left(\lambda^{+, \text {ho }}(\varepsilon), P^{+, \text {ho }}(\varepsilon)\right)$ для пары $(\lambda, P)$. Эти мажоранты строятся следуюшим образом. Во-первых, ограничимся значениями $\varepsilon$ такими, что

$$
V_{\mathrm{gl}}^{+}(1+\varepsilon)^{2}<\frac{(d-1)^{2}}{8}, \quad \varepsilon<\min \left[\frac{p_{k^{+}}\left(z^{+}\right)}{1-p_{k^{+}}\left(z^{+}\right)}, \frac{p_{\bar{k}^{+}}\left(w^{+}\right)}{1-p_{\bar{k}^{+}}\left(w^{+}\right)}\right] .
$$

Здесь $k^{+}=\min \left[k: p_{k}\left(z^{+}\right)>0\right]$ и $\bar{k}^{+}=\min \left[k: p_{k}\left(w^{+}\right)>0\right]$.

Далее, используя тот факт, что точка $w^{+}$является орисферическим максимумом, для данного $\varepsilon$ мы выберем точку $w(\varepsilon) \in \partial \mathbf{H}$ и орисферу $\mathbf{S}_{w(\varepsilon)} \in \mathfrak{G}_{w(\varepsilon)}^{-}$с полюсом $w(\varepsilon)$ такие, что для любого $z \in \mathbf{H} \backslash \mathbf{B}\left(\mathbf{S}_{w(\varepsilon)}\right)$

$$
\lambda(z) \leqslant \lambda\left(w^{+}\right)(1+\varepsilon) \quad \text { и } \quad p_{k}(z) \leqslant p_{k}\left(w^{+}\right)(1+\varepsilon) \quad \forall k \geqslant 2 .
$$

Наконец, положим для любого $z \in \mathbf{B}\left(\mathbf{S}_{w(\varepsilon)}\right)$

$$
\begin{aligned}
& {\left[\lambda^{+, \text {ho }}(\varepsilon)\right](z)=\lambda\left(z^{+}\right)(1+\varepsilon) \quad \text { и }} \\
& \left.p_{k}^{+, \text {ho }}(\varepsilon)\right](z)=p_{k}\left(z^{+}\right)(1+\varepsilon) \quad \forall k>k^{+}
\end{aligned}
$$

и для любого $z \in \mathbf{H} \backslash \mathbf{B}\left(\mathbf{S}_{w(\varepsilon)}\right)$

$$
\begin{aligned}
& {\left[\lambda^{+, \text {ho }}(\varepsilon)\right](z)=\lambda\left(w^{+}\right)(1+\varepsilon) \quad \text { и }} \\
& {\left[p_{k}^{+, \text {ho }}(\varepsilon)\right](z)=p_{k}\left(w^{+}\right)(1+\varepsilon) \quad \forall k>\bar{k}^{+} .}
\end{aligned}
$$

Мажоранта $\left(\lambda^{+}\right.$, ho $(\varepsilon), P^{+}$, ho $\left.(\varepsilon)\right)$ порождает верхний процесс $\Theta_{z_{0}}^{+ \text {, ho }}(\varepsilon)$, к которому применимы методы и результаты работы [4]. Из них вытекает невозвратность процесса $\Theta_{z_{0}}^{+, \text {ho }}(\varepsilon)$ и, следовательно, невозвратность исходного ВДП $\Theta_{z_{0}}$.

Далее, используя тот факт, что точка $w^{-}$является орисферическим минимумом, мы задаем семейство минорирующих пар $\left(\lambda^{- \text {,ho }}(\varepsilon)\right.$, 
$\left.P^{-, \text {ho }}(\varepsilon)\right)$, в точности так же, как и в $(4.2 \mathrm{a}),(4.2 \mathrm{~b})$. Как и ранее, они порождают нижние ВДП $\Theta_{z_{0}}^{-}(\varepsilon)$. Так же, как и в $(4.3),(4.4)$, мы получаем теперь, что

$$
\mathbb{P}_{z_{0}}^{\text {joint }}\left\{\Lambda_{z_{0}}^{-, \text {ho }}(\varepsilon) \subseteq \Lambda_{z_{0}} \subseteq \Lambda_{z_{0}}^{+, \text {ho }}(\varepsilon)\right\}=1
$$

и

$$
\mathbb{P}_{z_{0}}^{\text {joint }}\left\{h^{-, \text {ho }}\left(z_{0} ; \varepsilon\right) \leqslant h\left(z_{0}\right\} \leqslant h^{+, \text {ho }}\left(z_{0} ; \varepsilon\right)\right\}=1 \text {. }
$$

Здесь снова применимы методы и результаты работы [4], из которых вытекает, что п.н.

$$
\begin{aligned}
& h^{-, \text {ho }}\left(z_{0} ; \varepsilon\right)=\frac{d-1}{2}-\sqrt{\frac{(d-1)^{2}}{4}-V_{\mathrm{b}}^{-}(1-\varepsilon)^{2}}, \\
& h^{+, \text {ho }}\left(z_{0} ; \varepsilon\right)=\frac{d-1}{2}-\sqrt{\frac{(d-1)^{2}}{4}-V_{\mathrm{b}}^{+}(1+\varepsilon)^{2}} \text {. }
\end{aligned}
$$

Переходя к пределу при $\varepsilon \rightarrow 0$, получаем равенство (2.4) в теореме 2.A.

Проверка того, что в предположениях теоремы 2.А выполняется оценка (2.5) использует те же аргументы, что и в теореме 1.А. Единственное отличие в том, что вместо леммы 3.1 используется лемма 3.3 . Это завершает доказательство теоремы 2.А.

У'терждения Б и В в теореме 2 очевидны, и их доказательство опускается.

Доказательство теорем 3.А и 3.Б. Эти доказательства следуют той же схеме, что и доказательства теорем 1 и 2 . В них используются верхние и нижние процессы, порожденные ЛОО-мажорантами и ОГП-минорантами. Вместо лемм 3.1 и 3.2 используется лемма 3.4 в доказательстве теоремы 3.А и лемма 3.5 в доказательстве теоремы 3.Б.

\section{СПИСОК ЛИТЕРАТУРЫ}

1. Grigor'yan A., Kelbert $M$. Recurrence and transience of branching diffusion processes on Riemannian manifolds. - Ann. Probab., 2003, v. 31, № 1, p. 244-284.

2. Karpelevich F. I., Kelbert M. Ya., Suhov Yu. M. The boundedness of branching Markov processes. - The Dynkin Festschrift. Markov Processes and Their Applications. Ed. by M. Freidlin. Boston: Birkhäuser, 1994, p. 143-152. (Progr. Probab., v. 34.)

3. Karpelevich F.I., Pechersky E. A., Suhov Yu.M. A phase transition for hyperbolic branching processes. - Comm. Math. Phys., 1998, v. 195, № 3, p. 627-642.

4. Kelbert M. Ya., Suhov Yu.M. Branching diffusions on $\mathbf{H}^{d}$ with variable fission: The Hausdorff dimension of the limiting set. - Теория вероятн. и ее примен., 2006, T. 51 , B. 1 , c. $241-255$.

5. Lalley S., Sellke T. Hyperbolic branching Brownian motion. - Probab. Theory Relat. Fields, 1997, v. 108, № 2, p. 171-192.

6. Watson G. N. A Treatise on the Theory of Bessel Functions. Cambridge: Cambridge Univ. Press, 1966, 804 p. 